\title{
Infinitely many shape-invariant potentials and cubic identities of the Laguerre and Jacobi polynomials
}

\section{$\operatorname{AUTHOR}(\mathrm{S}):$}

Odake, S.; Sasaki, R.

\section{CITATION:}

Odake, S....[et al]. Infinitely many shape-invariant potentials and cubic identities of the Laguerre and Jacobi polynomials. Journal of Mathematical Physics 2010, 51(5): 053513.

\section{ISSUE DATE:}

2010-05-14

URL:

http://hdl.handle.net/2433/128858

RIGHT:

(C) 2010 American Institute of Physics 


\title{
Infinitely many shape-invariant potentials and cubic identities of the Laguerre and Jacobi polynomials
}

\author{
S. Odake ${ }^{1}$ and R. Sasaki ${ }^{2, a)}$ \\ ${ }^{1}$ Department of Physics, Shinshu University, Matsumoto 390-8621, Japan \\ ${ }^{2}$ Yukawa Institute for Theoretical Physics, Kyoto University, Kyoto 606-8502, Japan
}

(Received 12 November 2009; accepted 22 February 2010; published online 14 May 2010)

\begin{abstract}
We provide analytic proofs for the shape invariance of the recently discovered [Odake and Sasaki, Phys. Lett. B 679, 414 (2009)] two families of infinitely many exactly solvable one-dimensional quantum mechanical potentials. These potentials are obtained by deforming the well-known radial oscillator potential or the Darboux-Pöschl-Teller potential by a degree $\ell(\ell=1,2, \ldots)$ eigenpolynomial. The shape invariance conditions are attributed to new polynomial identities of degree $3 \ell$ involving cubic products of the Laguerre or Jacobi polynomials. These identities are proved elementarily by combining simple identities. (C) 2010 American Institute of Physics. [doi:10.1063/1.3371248]
\end{abstract}

\section{INTRODUCTION}

In a previous letter, ${ }^{1}$ two sets of infinitely many shape-invariant ${ }^{2}$ potentials were derived by deforming the radial oscillator potential ${ }^{3,4}$ and the Darboux-Pöschl-Teller (DPT) potential ${ }^{5,6}$ in terms of a polynomial eigenfunction of degree $\ell(\ell=1,2, \ldots)$. As the main part of the eigenfunctions of these exactly solvable quantum mechanical systems, the exceptional $\left(X_{\ell}\right)$ Laguerre and Jacobi polynomials were obtained. ${ }^{1}$ The lowest $(\ell=1)$ examples, the $X_{1}$ Laguerre and Jacobi polynomials, are equivalent to those introduced in the pioneering work of Gomez-Ullate $e t$ al. ${ }^{7,8}$ within the Sturm-Liouville theory. The reformulation in the framework of quantum mechanics and shape-invariant potentials was done by Quesne et al. ${ }^{9,10}$ By construction these new orthogonal polynomials satisfy a second order differential equation (the Schrödinger equation) without contradicting Bochner's theorem, ${ }^{11}$ since they start at degree $\ell,(\ell=1,2, \ldots)$ instead of the degree zero constant term.

Here we present analytical proofs of the main assertion that these deformed potentials are indeed shape invariant, which could not be given in the letter ${ }^{1}$ due to the lack of space. For both the deformed radial oscillator potential and deformed trigonometric/hyperbolic DPT potentials, the condition for shape invariance for each $\ell$ is satisfied if a certain identity involving cubic products of the Laguerre or Jacobi polynomials holds. To the best of our knowledge, these infinitely many identities were not presented before. We show that these identities are derived by combining several elementary relations among the Laguerre or Jacobi polynomials.

This paper is organized as follows. In Sec. II the general setting of shape invariance and the deformation of a potential in terms of a polynomial eigenfunction is recapitulated together with many preparatory materials. In Sec. III we show that the condition for shape invariance for each $\ell$ is attributed to a certain polynomial identity of degree $3 \ell$ involving cubic products of the Laguerre or Jacobi polynomials of various parameters, (3.4) and (3.11). Then we show that these identities are simply derived by combining simple relations among the Laguerre polynomials (3.5) and (3.6) and Jacobi polynomials (3.12) and (3.13). Section IV is for a summary and comments on related results to be published in a forthcoming paper.

\footnotetext{
${ }^{a)}$ Electronic mail: ryu@yukawa.kyoto-u.ac.jp.
} 


\section{GENERAL SETTING}

As in Ref. 1, let us start with a generic one-dimensional quantum mechanical system in a factorized form,

$$
\begin{gathered}
\mathcal{H}=\mathcal{A}^{\dagger} \mathcal{A}=p^{2}+U(x), \quad p=-i \partial_{x}, \quad U(x)=\left(\partial_{x} w(x)\right)^{2}+\partial_{x}^{2} w(x), \\
\mathcal{A} \stackrel{\operatorname{def}}{=} \partial_{x}-\partial_{x} w(x), \quad \mathcal{A}^{\dagger}=-\partial_{x}-\partial_{x} w(x) .
\end{gathered}
$$

Here we call a real and smooth function $w(x)$ a prepotential. It parametrizes the ground state wave function $\phi_{0}(x)$, which has no node and can be chosen real and positive, $\phi_{0}(x)=e^{w(x)}$. It is trivial to verify $\mathcal{A} \phi_{0}(x)=0$ and $\mathcal{H} \phi_{0}(x)=0$.

Shape invariance is realized by specific dependence of the potential, or the prepotential on a set of parameters $\boldsymbol{\lambda}=\left(\lambda_{1}, \lambda_{2}, \ldots\right)$, to be denoted by $w(x ; \boldsymbol{\lambda}), \mathcal{A}(\boldsymbol{\lambda}), \mathcal{H}(\boldsymbol{\lambda}), \mathcal{E}_{n}(\boldsymbol{\lambda})$, etc. The shape invariance condition to be discussed in this paper is written simply

$$
\begin{gathered}
\mathcal{A}(\boldsymbol{\lambda}) \mathcal{A}(\boldsymbol{\lambda})^{\dagger}=\mathcal{A}(\boldsymbol{\lambda}+\boldsymbol{\delta})^{\dagger} \mathcal{A}(\boldsymbol{\lambda}+\boldsymbol{\delta})+\mathcal{E}_{1}(\boldsymbol{\lambda}), \\
\text { or } \quad\left(\partial_{x} w(x ; \boldsymbol{\lambda})\right)^{2}-\partial_{x}^{2} w(x ; \boldsymbol{\lambda})=\left(\partial_{x} w(x ; \boldsymbol{\lambda}+\boldsymbol{\delta})\right)^{2}+\partial_{x}^{2} w(x ; \boldsymbol{\lambda}+\boldsymbol{\delta})+\mathcal{E}_{1}(\boldsymbol{\lambda}),
\end{gathered}
$$

in which $\boldsymbol{\delta}$ is a certain shift of the parameters. Then the entire set of discrete eigenvalues and the corresponding eigenfunctions of $\mathcal{H}=\mathcal{H}(\boldsymbol{\lambda})$,

$$
\begin{gathered}
\mathcal{H}(\boldsymbol{\lambda}) \phi_{n}(x ; \boldsymbol{\lambda})=\mathcal{E}_{n}(\boldsymbol{\lambda}) \phi_{n}(x ; \boldsymbol{\lambda}), \quad n=0,1,2, \ldots, \\
\phi_{n}(x ; \boldsymbol{\lambda})=\phi_{0}(x ; \boldsymbol{\lambda}) P_{n}(\eta(x) ; \boldsymbol{\lambda}),
\end{gathered}
$$

is determined algebraically, ${ }^{2,4,12,13}$

$$
\begin{gathered}
\mathcal{E}_{n}(\boldsymbol{\lambda})=\sum_{k=0}^{n-1} \mathcal{E}_{1}(\boldsymbol{\lambda}+k \boldsymbol{\delta}), \\
\phi_{n}(x ; \boldsymbol{\lambda}) \propto \mathcal{A}(\boldsymbol{\lambda})^{\dagger} \mathcal{A}(\boldsymbol{\lambda}+\boldsymbol{\delta})^{\dagger} \cdots \mathcal{A}(\boldsymbol{\lambda}+(n-1) \boldsymbol{\delta})^{\dagger} \times e^{w(x ; \boldsymbol{\lambda}+n \boldsymbol{\delta})} .
\end{gathered}
$$

The polynomial eigenfunction $P_{n}(\eta(x) ; \boldsymbol{\lambda})$, which is the Laguerre or Jacobi polynomial in $\eta(x)$, satisfies

$$
-\partial_{x}^{2} P_{n}(\eta(x) ; \boldsymbol{\lambda})-2 \partial_{x} w(x ; \boldsymbol{\lambda}) \partial_{x} P_{n}(\eta(x) ; \boldsymbol{\lambda})=\mathcal{E}_{n}(\boldsymbol{\lambda}) P_{n}(\eta(x) ; \boldsymbol{\lambda}) .
$$

Here $\eta(x)$ is a function of $x$ called the sinusoidal coordinate. ${ }^{14}$

In Ref. 1 a shape-invariant prepotential $w(x ; \boldsymbol{\lambda})=w_{0}(x ; \boldsymbol{\lambda})$ is deformed by a polynomial eigenfunction $\xi_{\ell}$ of its Hamiltonian to produce another shape-invariant prepotential $w_{\ell}(\ell=1,2, \ldots)$,

$$
w_{\ell}(x ; \boldsymbol{\lambda})=w_{0}(x ; \boldsymbol{\lambda}+\ell \boldsymbol{\delta})+\log \frac{\xi_{\ell}(\eta(x) ; \boldsymbol{\lambda}+\boldsymbol{\delta})}{\xi_{\ell}(\eta(x) ; \boldsymbol{\lambda})},
$$

in which $\xi_{\ell}$ is related to the polynomial eigenfunction $P_{n}$ above (2.6). It should be noted that the normalization of the polynomial $\xi_{\ell}(x ; \boldsymbol{\lambda})$ is irrelevant to the deformation. The $\ell=0$ case corresponds to the original system. The $\ell$ th Hamiltonian and eigenfunctions, etc., are given by

$$
\begin{gathered}
\mathcal{A}_{\ell}(\boldsymbol{\lambda}) \stackrel{\text { def }}{=} \partial_{x}-\partial_{x} w_{\ell}(x ; \boldsymbol{\lambda}), \quad \mathcal{A}_{\ell}(\boldsymbol{\lambda})^{\dagger}=-\partial_{x}-\partial_{x} w_{\ell}(x ; \boldsymbol{\lambda}), \\
\mathcal{H}_{\ell}(\boldsymbol{\lambda}) \stackrel{\operatorname{def}}{=} \mathcal{A}_{\ell}(\boldsymbol{\lambda})^{\dagger} \mathcal{A}_{\ell}(\boldsymbol{\lambda}),
\end{gathered}
$$




$$
\begin{gathered}
\mathcal{H}_{\ell}(\boldsymbol{\lambda}) \phi_{\ell, n}(x ; \boldsymbol{\lambda})=\mathcal{E}_{n}(\boldsymbol{\lambda}+\ell \boldsymbol{\delta}) \phi_{\ell, n}(x ; \boldsymbol{\lambda}), \\
\phi_{\ell, n}(x ; \boldsymbol{\lambda})=\psi_{\ell}(x ; \boldsymbol{\lambda}) P_{\ell, n}(\eta(x) ; \boldsymbol{\lambda}), \quad \psi_{\ell}(x ; \boldsymbol{\lambda})=\frac{\operatorname{def}}{\xi_{\ell}(\eta(x) ; \boldsymbol{\lambda})} .
\end{gathered}
$$

The orthogonality of the eigenfunctions of the Hamiltonian $\mathcal{H}_{\ell}(\boldsymbol{\lambda})$ reads

$$
\int \psi_{\ell}(x ; \boldsymbol{\lambda})^{2} P_{\ell, n}(\eta(x) ; \boldsymbol{\lambda}) P_{\ell, m}(\eta(x) ; \boldsymbol{\lambda}) d x=h_{\ell, n}(\boldsymbol{\lambda}) \delta_{n m}, \quad h_{\ell, n}(\boldsymbol{\lambda})>0,
$$

in which $\psi_{\ell}(x ; \boldsymbol{\lambda})^{2}$ is the orthogonality measure and $P_{\ell, n}(x ; \boldsymbol{\lambda})$ is the $n$th member of the exceptional $\left(X_{\ell}\right)$ orthogonal polynomial. It is expressed in terms of $\xi_{\ell}(x)$ 's and $P_{n}(x)$ 's as shown in (2.22), (2.27), and (2.34).

In this paper we will demonstrate that the deformed prepotential $w_{\ell}(\ell=1,2, \ldots)$ actually satisfies the shape invariance condition,

$$
\Delta_{\ell}(x ; \boldsymbol{\lambda})=0,
$$

in which $\Delta_{\ell}(x ; \boldsymbol{\lambda})$ is defined by

$$
\Delta_{\ell}(x ; \boldsymbol{\lambda})=\left(\partial_{x} w_{\ell}(x ; \boldsymbol{\lambda})\right)^{2}-\partial_{x}^{2} w_{\ell}(x ; \boldsymbol{\lambda})-\left(\partial_{x} w_{\ell}(x ; \boldsymbol{\lambda}+\boldsymbol{\delta})\right)^{2}-\partial_{x}^{2} w_{\ell}(x ; \boldsymbol{\lambda}+\boldsymbol{\delta})-\mathcal{E}_{1}(\boldsymbol{\lambda}+\ell \boldsymbol{\delta}),
$$

for the three cases, the radial oscillator and the trigonometric/hyperbolic DPT discussed in Ref. 1. The proof consists of two steps. First in Sec. III we transform, by utilizing the differential equations for $\xi_{\ell}(\eta(x) ; \boldsymbol{\lambda}),(2.41)$, etc, the shape invariance condition (2.17) into an identity involving products of three Laguerre or Jacobi polynomials of various parameters, (3.4) and (3.11). The trigonometric and hyperbolic DPTs lead to the same identities. Second these cubic identities are proven by combining simple identities among the Laguerre or Jacobi polynomials of neighboring degrees $n, n-1$ and parameters $\alpha, \alpha \pm 1, \beta, \beta \pm 1$.

Here we show various data necessary for the proof. They are recapitulated from Ref. 1.

(1) Radial oscillator:

$$
\begin{aligned}
& \stackrel{\text { def }}{=} g, \quad \boldsymbol{\delta}=1, \quad g>0, \\
& \mathcal{E}_{n}(\boldsymbol{\lambda})=4 n, \quad \eta(x)^{\mathrm{def}}=x^{2}, \quad 0<x<\infty, \\
& \phi_{0}(x ; \boldsymbol{\lambda})=e^{-x^{2} / 2} x^{g} \Leftrightarrow w_{0}(x ; \boldsymbol{\lambda})=-\frac{x^{2}}{2}+g \log x, \\
& P_{n}(x ; \boldsymbol{\lambda})=L_{n}^{\text {def }}(g-1 / 2)(x), \quad \xi_{\ell}(x ; \boldsymbol{\lambda})=L_{\ell}^{(g+\ell-3 / 2)}(-x), \\
& P_{\ell, n}(x ; \boldsymbol{\lambda})=\xi_{\ell}(x ; g+1) P_{n}(x ; g+\ell)-\xi_{\ell-1}(x ; g+2) P_{n-1}(x ; g+\ell) .
\end{aligned}
$$

(2) Trigonometric DPT:

$$
\begin{gathered}
\text { def } \\
\boldsymbol{\lambda}=(g, h), \quad \boldsymbol{\delta}=(1,1), \quad h>g>0, \\
\mathcal{E}_{n}(\boldsymbol{\lambda})=4 n(n+g+h), \quad \eta(x)=\cos 2 x, \quad 0<x<\frac{\pi}{2},
\end{gathered}
$$




$$
\begin{gathered}
\phi_{0}(x ; \boldsymbol{\lambda})=(\sin x)^{g}(\cos x)^{h} \Leftrightarrow w_{0}(x ; \boldsymbol{\lambda})=g \log \sin x+h \log \cos x, \\
P_{n}(x ; \boldsymbol{\lambda})=P_{n}^{(g-1 / 2, h-1 / 2)}(x), \quad \xi_{\ell}(x ; \boldsymbol{\lambda})=P_{\ell}^{(-g-\ell-1 / 2, h+\ell-3 / 2)}(x), \\
P_{\ell, n}(x ; \boldsymbol{\lambda})=a_{\ell, n}(x ; \boldsymbol{\lambda}) P_{n}(x ; \boldsymbol{\lambda}+\ell \boldsymbol{\delta})+b_{\ell, n}(x ; \boldsymbol{\lambda}) P_{n-1}(x ; \boldsymbol{\lambda}+\ell \boldsymbol{\delta}), \\
a_{\ell, n}(x ; \boldsymbol{\lambda})=\xi_{\ell}(x ; g+1, h+1)+\frac{2 n(-g+h+\ell-1)}{(-g+h+2 \ell-2)(g+h+2 n+2 \ell-1)} \xi_{\ell-1}(x ; g, h+2) \\
-\frac{n(2 h+4 \ell-3) \xi_{\ell-2}(x ; g+1, h+3)}{(2 g+2 n+1)(-g+h+2 \ell-2)}, \\
b_{\ell, n}(x ; \boldsymbol{\lambda})=\frac{\operatorname{def}(-g+h+\ell-1)(2 g+2 n+2 \ell-1)}{(2 g+2 n+1)(g+h+2 n+2 \ell-1)} \xi_{\ell-1}(x ; g, h+2) .
\end{gathered}
$$

(3) Hyperbolic DPT:

$$
\begin{aligned}
& \boldsymbol{\lambda}=(g, h), \quad \boldsymbol{\delta}=(1,-1), \quad h>g>0, \quad \ell<n_{B}=\left[\frac{\operatorname{def}}{2}(h-g)\right]^{\prime}, \\
& \mathcal{E}_{n}(\boldsymbol{\lambda})=4 n(h-g-n), \quad \eta(x)=\cosh 2 x, \quad 0<x<\infty, \\
& \phi_{0}(x ; \boldsymbol{\lambda}) \stackrel{\operatorname{def}}{=}(\sinh x)^{g}(\cosh x)^{-h} \Leftrightarrow w_{0}(x ; \boldsymbol{\lambda})=g \log \sinh x-h \log \cosh x, \\
& P_{n}(x ; \boldsymbol{\lambda}) \stackrel{\text { def }}{=} P_{n}^{(g-1 / 2,-h-1 / 2)}(x), \quad \xi_{\ell}(x ; \boldsymbol{\lambda}) \stackrel{\operatorname{def}}{=} P_{\ell}^{(-g-\ell-1 / 2,-h+\ell-3 / 2)}(x), \\
& P_{\ell, n}(x ; \boldsymbol{\lambda}) \stackrel{\text { def }}{=} a_{\ell, n}(x ; \boldsymbol{\lambda}) P_{n}(x ; \boldsymbol{\lambda}+\ell \boldsymbol{\delta})+b_{\ell, n}(x ; \boldsymbol{\lambda}) P_{n-1}(x ; \boldsymbol{\lambda}+\ell \boldsymbol{\delta}), \\
& a_{\ell, n}(x ; \boldsymbol{\lambda}) \stackrel{\operatorname{def}}{=} \xi_{\ell}(x ; g+1, h-1)+\frac{2 n(-g-h+\ell-1) \xi_{\ell-1}(x ; g, h-2)}{(-g-h+2 \ell-2)(g-h+2 n+2 \ell-1)} \\
& -\frac{n(-2 h+4 \ell-3) \xi_{\ell-2}(x ; g+1, h-3)}{(2 g+2 n+1)(-g-h+2 \ell-2)}, \\
& b_{\ell, n}(x ; \boldsymbol{\lambda})=\frac{\operatorname{def}(-g-h+\ell-1)(2 g+2 n+2 \ell-1)}{(2 g+2 n+1)(g-h+2 n+2 \ell-1)} \xi_{\ell-1}(x ; g, h-2) .
\end{aligned}
$$

In (2.30) $[x]^{\prime}$ denotes the greatest integer not equal or exceeding $x$. Here $L_{n}^{(\alpha)}(x)$ is the Laguerre polynomial and $P_{n}^{(\alpha, \beta)}(x)$ is the Jacobi polynomial. The polynomial eigenfunction $P_{\ell, n}(x ; \boldsymbol{\lambda})(2.22)$, (2.27), or (2.34) is a degree $\ell+n$ polynomial in $x$. In a future publication ${ }^{15}$ we will present equivalent forms of the $X_{\ell}$ polynomials $P_{\ell, n}(x ; \boldsymbol{\lambda})$ which appear much simpler than those given in (2.22), (2.27), and (2.34). Needless to say that the deforming polynomials $\xi_{\ell}(\eta(x) ; \boldsymbol{\lambda})$ and $\xi_{\ell}(\eta(x) ; \boldsymbol{\lambda}+\boldsymbol{\delta})$ are of the same sign in the domain for all the three cases. In other words, the deforming polynomials do not have a zero in the domains listed in (2.19), (2.24), and (2.31), respectively. To see this we use the expansion formula of the Laguerre and Jacobi polynomials,

$$
L_{n}^{(\alpha)}(x)=\frac{1}{n !} \sum_{k=0}^{n} \frac{(-n)_{k}}{k !}(\alpha+k+1)_{n-k} x^{k}
$$




$$
P_{n}^{(\alpha, \beta)}(x)=\frac{(\alpha+1)_{n}}{n !} \sum_{k=0}^{n} \frac{1}{k !} \frac{(-n)_{k}(n+\alpha+\beta+1)_{k}}{(\alpha+1)_{k}}\left(\frac{1-x}{2}\right)^{k} .
$$

For the radial oscillator we obtain

$$
\xi_{\ell}(\eta(x) ; \boldsymbol{\lambda})=\sum_{k=0}^{\ell} \frac{\left(g+\ell+k-\frac{1}{2}\right)_{\ell-k}}{k !(\ell-k) !} x^{2 k}>0,
$$

and for the trigonometric/hyperbolic DPT,

$$
(-1)^{\ell} \xi_{\ell}(\eta(x) ; \boldsymbol{\lambda})=\left\{\begin{array}{c}
\frac{(g+1 / 2)_{\ell}}{\ell !} \sum_{k=0}^{\ell} \frac{(\ell-k+1)_{k}(h-g+\ell-1)_{k}}{k !\left(g+\ell-k+\frac{1}{2}\right)_{k}}(\sin x)^{2 k}>0 \\
\frac{(g+1 / 2)_{\ell}}{\ell !} \sum_{k=0}^{\ell} \frac{(\ell-k+1)_{k}(g+h+2-\ell-k)_{k}}{k !\left(g+\ell-k+\frac{1}{2}\right)_{k}}(\sinh x)^{2 k}>0 .
\end{array}\right.
$$

because each term in summation is positive. This guarantees the positive definiteness of the orthogonality measure $\psi_{\ell}(x ; \boldsymbol{\lambda})^{2}(2.14)$ and the singularity free structure as well as the Hermiticity (self-adjointness) of the Hamiltonian. The oscillation theorem for the one-dimensional quantum mechanical systems dictates that the $n$th excited state polynomial eigenfunction $P_{\ell, n}(\eta(x) ; \boldsymbol{\lambda})$ has $n$ zeros in the domain (2.19), (2.24), or (2.31), although it is a degree $\ell+n$ polynomial in $\eta$.

Let us note that the terms of the form of the complete square $\left(\partial_{x} \xi_{\ell}(\eta(x) ; \boldsymbol{\lambda}) / \xi_{\ell}(\eta(x) ; \boldsymbol{\lambda})\right)^{2}$, $\left(\partial_{x} \xi_{\ell}(\eta(x) ; \boldsymbol{\lambda}+\boldsymbol{\delta}) / \xi_{\ell}(\eta(x) ; \boldsymbol{\lambda}+\boldsymbol{\delta})\right)^{2}$, and $\left(\partial_{x} \xi_{\ell}(\eta(x) ; \boldsymbol{\lambda}+2 \boldsymbol{\delta}) / \xi_{\ell}(\eta(x) ; \boldsymbol{\lambda}+2 \boldsymbol{\delta})\right)^{2}$, cancel out in $\Delta_{\ell}(x ; \boldsymbol{\lambda})$ (2.17). Then we use the fact that, corresponding to (2.9), the deforming polynomial $\xi_{\ell}(\eta(x) ; \boldsymbol{\lambda})$ also satisfies a second order linear differential equation,

$$
-\partial_{x}^{2} \xi_{\ell}(\eta(x) ; \boldsymbol{\lambda})-2 \partial_{x} \widetilde{w}_{0}(x ; \boldsymbol{\lambda}, \ell) \partial_{x} \xi_{\ell}(\eta(x) ; \boldsymbol{\lambda})=\widetilde{\mathcal{E}}_{\ell}(\boldsymbol{\lambda}) \xi_{\ell}(\eta(x) ; \boldsymbol{\lambda}),
$$

where $\widetilde{w}_{0}(x ; \boldsymbol{\lambda}, \ell)$ and $\widetilde{\mathcal{E}}_{\ell}(\boldsymbol{\lambda})$ are

$$
\begin{gathered}
\widetilde{w}_{0}(x ; \boldsymbol{\lambda}, \ell)= \begin{cases}\frac{1}{2} x^{2}+(g+\ell-1) \log x & \text { :radial osci. } \\
w_{0}(x ;-g-\ell, h+\ell-1) & \text { :trig.DPT } \\
w_{0}(x ;-g-\ell, h-\ell+1) & \text { :hyper.DPT },\end{cases} \\
\widetilde{\mathcal{E}}_{\ell}(\boldsymbol{\lambda})= \begin{cases}-4 \ell & \text { :radial osci. } \\
\mathcal{E}_{\ell}(-g-\ell, h+\ell-1) & \text { :trig.DPT } \\
\mathcal{E}_{\ell}(-g-\ell, h-\ell+1) & \text { :hyper.DPT. }\end{cases}
\end{gathered}
$$

Substituting (2.10) into (2.17) and using the shape invariance of the undeformed system $\Delta_{0}(x ; \boldsymbol{\lambda}$ $+\ell \boldsymbol{\delta})=0$ and $(2.41)$, we obtain

$$
\begin{aligned}
\Delta_{\ell}(x ; \boldsymbol{\lambda}) & \times \frac{1}{2} \xi_{\ell}(\eta ; \boldsymbol{\lambda}) \xi_{\ell}(\eta ; \boldsymbol{\lambda}+\boldsymbol{\delta}) \xi_{\ell}(\eta ; \boldsymbol{\lambda}+2 \boldsymbol{\delta}) \\
= & \partial_{x} \widetilde{w}_{0}(x ; \boldsymbol{\lambda}+2 \delta, \ell) \partial_{x} \xi_{\ell}(\eta ; \boldsymbol{\lambda}+2 \boldsymbol{\delta}) \xi_{\ell}(\eta ; \boldsymbol{\lambda}) \xi_{\ell}(\eta ; \boldsymbol{\lambda}+\boldsymbol{\delta}) \\
& -\partial_{x} \widetilde{w}_{0}(x ; \boldsymbol{\lambda}, \ell) \partial_{x} \xi_{\ell}(\eta ; \boldsymbol{\lambda}) \xi_{\ell}(\eta ; \boldsymbol{\lambda}+\boldsymbol{\delta}) \xi_{\ell}(\eta ; \boldsymbol{\lambda}+2 \boldsymbol{\delta})
\end{aligned}
$$




$$
\begin{aligned}
& +\frac{1}{2}\left(\widetilde{\mathcal{E}}_{\ell}(\boldsymbol{\lambda}+2 \boldsymbol{\delta})-\widetilde{\mathcal{E}}_{\ell}(\boldsymbol{\lambda})\right) \xi_{\ell}(\eta ; \boldsymbol{\lambda}) \xi_{\ell}(\eta ; \boldsymbol{\lambda}+\boldsymbol{\delta}) \xi_{\ell}(\eta ; \boldsymbol{\lambda}+2 \boldsymbol{\delta}) \\
& +\partial_{x} w_{0}(x ; \boldsymbol{\lambda}+\ell \boldsymbol{\delta})\left(\partial_{x} \xi_{\ell}(\eta ; \boldsymbol{\lambda}+\boldsymbol{\delta}) \xi_{\ell}(\eta ; \boldsymbol{\lambda})-\partial_{x} \xi_{\ell}(\eta ; \boldsymbol{\lambda}) \xi_{\ell}(\eta ; \boldsymbol{\lambda}+\boldsymbol{\delta})\right) \xi_{\ell}(\eta ; \boldsymbol{\lambda}+2 \boldsymbol{\delta}) \\
& -\partial_{x} w_{0}(x ; \boldsymbol{\lambda}+\ell \boldsymbol{\delta}+\boldsymbol{\delta})\left(\partial_{x} \xi_{\ell}(\eta ; \boldsymbol{\lambda}+2 \boldsymbol{\delta}) \xi_{\ell}(\eta ; \boldsymbol{\lambda}+\boldsymbol{\delta})-\partial_{x} \xi_{\ell}(\eta ; \boldsymbol{\lambda}+\boldsymbol{\delta}) \xi_{\ell}(\eta ; \boldsymbol{\lambda}+2 \boldsymbol{\delta})\right) \xi_{\ell}(\eta ; \boldsymbol{\lambda}) \\
& -\partial_{x} \xi_{\ell}(\eta ; \boldsymbol{\lambda}) \partial_{x} \xi_{\ell}(\eta ; \boldsymbol{\lambda}+\boldsymbol{\delta}) \xi_{\ell}(\eta ; \boldsymbol{\lambda}+2 \boldsymbol{\delta})+\partial_{x} \xi_{\ell}(\eta ; \boldsymbol{\lambda}+\boldsymbol{\delta}) \partial_{x} \xi_{\ell}(\eta ; \boldsymbol{\lambda}+2 \boldsymbol{\delta}) \xi_{\ell}(\eta ; \boldsymbol{\lambda}),
\end{aligned}
$$

where $\eta=\eta(x)$. It is essential that the right hand side of (2.44) is now a polynomial in $\eta(x)$ because $\partial_{x} \eta(x) \partial_{x} w_{0}$ and $\partial_{x} \eta(x) \partial_{x} \widetilde{w}_{0}$ and $\left(\partial_{x} \eta(x)\right)^{2}$ are expressed by $\eta(x)$,

$$
\begin{gathered}
\partial_{x} \eta(x) \partial_{x} w_{0}(x ; \boldsymbol{\lambda})=2 \times \begin{cases}g-\eta(x) & \text { :radial osci. } \\
-(g-h+(g+h) \eta(x)) & \text { :trig.DPT } \\
g+h+(g-h) \eta(x) & \text { :hyper.DPT, }\end{cases} \\
\partial_{x} \eta(x) \partial_{x} \widetilde{w}_{0}(x ; \boldsymbol{\lambda}, \ell)=2 \times \begin{cases}g+\ell-1+\eta(x) & \text { :radial osci. } \\
g+h+2 \ell-1+(g-h+1) \eta(x) & \text { :trig.DPT } \\
-(g-h+2 \ell-1+(g+h+1) \eta(x)) & \text { :hyper.DPT, }\end{cases} \\
\left(\partial_{x} \eta(x)\right)^{2}=4 \times \begin{cases}\eta(x) & \text { :radial osci. } \\
1-\eta(x)^{2} & \text { :trig.DPT } \\
-\left(1-\eta(x)^{2}\right) & \text { :hyper.DPT. }\end{cases}
\end{gathered}
$$

\section{CUBIC IDENTITIES}

The conditions for the shape invariance (2.16) are shown to be equivalent to cubic identities involving the Laguerre or Jacobi polynomials.

\section{A. Radial oscillator}

We fix $\ell$ and use a new parameter $\alpha$ instead of $g$,

$$
\begin{gathered}
\operatorname{def} \\
\alpha=g+\ell-\frac{1}{2} .
\end{gathered}
$$

By using the forward shift relation for the Laguerre polynomial,

$$
\partial_{x} L_{n}^{(\alpha)}(x)=-L_{n-1}^{(\alpha+1)}(x),
$$

the polynomial $\xi_{\ell}$ and its derivative are expressed as

$$
\xi_{\ell}(\eta ; \boldsymbol{\lambda})=L_{\ell}^{(\alpha-1)}(-\eta), \quad \partial_{x} \xi_{\ell}(\eta ; \boldsymbol{\lambda})=\partial_{x} \eta L_{\ell-1}^{(\alpha)}(-\eta),
$$

where $\eta=\eta(x)$. After replacing $-\eta(x)$ with $x$ and dividing by 4 , the condition for the shape invariance (2.16) with (2.44) is transformed into the following polynomial identity of degree $3 \ell$ in $x$ which contains products of three Laguerre polynomials of various parameters:

$$
\begin{aligned}
0= & -x L_{\ell-1}^{(\alpha+2)}(x) L_{\ell}^{(\alpha-1)}(x) L_{\ell}^{(\alpha)}(x)-\alpha L_{\ell-1}^{(\alpha)}(x) L_{\ell}^{(\alpha+1)}(x) L_{\ell}^{(\alpha)}(x)+(x+\alpha+1) L_{\ell-1}^{(\alpha+1)}(x) L_{\ell}^{(\alpha+1)}(x) L_{\ell}^{(\alpha-1)}(x) \\
& +x L_{\ell-1}^{(\alpha)}(x) L_{\ell-1}^{(\alpha+1)}(x) L_{\ell}^{(\alpha+1)}(x)-x L_{\ell-1}^{(\alpha+1)}(x) L_{\ell-1}^{(\alpha+2)}(x) L_{\ell}^{(\alpha-1)}(x) .
\end{aligned}
$$

To the best of our knowledge, this identity has not been reported before. For $\ell=0$ this identity is trivial, since $L_{-1}^{(\alpha)}(x)=0$. For lower $\ell$ it can be easily verified by direct calculation.

Below we will prove the identity (3.4) for an arbitrary positive integer $\ell$ by combining a few elementary relations among the Laguerre polynomials of neighboring degrees $n$ and $n-1$ and neighboring parameters $\alpha, \alpha \pm 1$, 


$$
\begin{gathered}
\text { Lemma (A) } L_{n}^{(\alpha-1)}(x)+L_{n-1}^{(\alpha)}(x)=L_{n}^{(\alpha)}(x), \\
\text { Lemma (B) } x L_{n-1}^{(\alpha+1)}(x)-\alpha L_{n-1}^{(\alpha)}(x)=-n L_{n}^{(\alpha-1)}(x),
\end{gathered}
$$

which can be verified elementarily based on the expansion formula of the Laguerre polynomial (2.37). The right hand side of the identity (3.4) can be written as

$$
\begin{aligned}
& \text { r.h.s. of }(3.4)=-x L_{\ell-1}^{(\alpha+2)}(x) L_{\ell}^{(\alpha-1)}(x)\left\{L_{\ell}^{(\alpha)}(x)+L_{\ell-1}^{(\alpha+1)}(x)\right\} \\
& +x L_{\ell-1}^{(\alpha+1)}(x) L_{\ell}^{(\alpha+1)}(x)\left\{L_{\ell}^{(\alpha-1)}(x)+L_{\ell-1}^{(\alpha)}(x)\right\} \\
& +L_{\ell}^{(\alpha+1)}(x)\left((\alpha+1) L_{\ell-1}^{(\alpha+1)}(x) L_{\ell}^{(\alpha-1)}(x)-\alpha L_{\ell-1}^{(\alpha)}(x) L_{\ell}^{(\alpha)}(x)\right) \\
& =L_{\ell}^{(\alpha+1)}(x)\left(L_{\ell}^{(\alpha)}(x)\left\{x L_{\ell-1}^{(\alpha+1)}(x)-\alpha L_{\ell-1}^{(\alpha)}(x)\right\}\right. \\
& \left.+L_{\ell}^{(\alpha-1)}(x)\left\{-x L_{\ell-1}^{(\alpha+2)}(x)+(\alpha+1) L_{\ell-1}^{(\alpha+1)}(x)\right\}\right) \\
& =L_{\ell}^{(\alpha+1)}(x)\left(L_{\ell}^{(\alpha)}(x)\left(-\ell L_{\ell}^{(\alpha-1)}(x)\right)+L_{\ell}^{(\alpha-1)}(x)\left(+\ell L_{\ell}^{(\alpha)}(x)\right)\right)=0 .
\end{aligned}
$$

Here we have used Lemma (A) in the first two curly brackets $\{\cdots\}$ and Lemma (B) in the next two curly brackets. This concludes the proof of the identity (3.4).

\section{B. Trigonometric DPT}

We fix $\ell$ and use new parameters $\alpha$ and $\beta$ instead of $g$ and $h$,

$$
\alpha=-g-\ell-\frac{1}{2}, \quad \beta=h+\ell-\frac{3}{2} .
$$

By using the forward shift relation for the Jacobi polynomial,

$$
\partial_{x} P_{n}^{(\alpha, \beta)}(x)=\frac{1}{2}(n+\alpha+\beta+1) P_{n-1}^{(\alpha+1, \beta+1)}(x),
$$

the polynomial $\xi_{\ell}$ and its derivative are expressed as

$$
\xi_{\ell}(\eta ; \boldsymbol{\lambda})=P_{\ell}^{(\alpha, \beta)}(\eta), \quad \partial_{x} \xi_{\ell}(\eta ; \boldsymbol{\lambda})=\partial_{x} \eta \frac{1}{2}(\ell+\alpha+\beta+1) P_{\ell-1}^{(\alpha+1, \beta+1)}(\eta),
$$

where $\eta=\eta(x)$. The condition for the shape invariance (2.16) with (2.44), after replacing $\eta(x)$ with $x$ and dividing by $-(\ell+\alpha+\beta+1)$, is simplified to a polynomial identity of degree $3 \ell$, which contains products of three Jacobi polynomials of various parameters,

$$
\begin{aligned}
0= & 2(\alpha-1)(1+x) P_{\ell-1}^{(\alpha-1, \beta+3)}(x) P_{\ell}^{(\alpha, \beta)}(x) P_{\ell}^{(\alpha-1, \beta+1)}(x) \\
& +2(\beta+1)(1-x) P_{\ell-1}^{(\alpha+1, \beta+1)}(x) P_{\ell}^{(\alpha-2, \beta+2)}(x) P_{\ell}^{(\alpha-1, \beta+1)}(x) \\
& -2(\alpha(1+x)+(\beta+2)(1-x)) P_{\ell-1}^{(\alpha, \beta+2)}(x) P_{\ell}^{(\alpha, \beta)}(x) P_{\ell}^{(\alpha-2, \beta+2)}(x) \\
& +(\ell+\alpha+\beta+1)\left(1-x^{2}\right) P_{\ell-1}^{(\alpha+1, \beta+1)}(x) P_{\ell-1}^{(\alpha, \beta+2)}(x) P_{\ell}^{(\alpha-2, \beta+2)}(x) \\
& -(\ell+\alpha+\beta+1)\left(1-x^{2}\right) P_{\ell-1}^{(\alpha, \beta+2)}(x) P_{\ell-1}^{(\alpha-1, \beta+3)}(x) P_{\ell}^{(\alpha, \beta)}(x) .
\end{aligned}
$$

To the best of our knowledge, this identity has not been reported before, either. For $\ell=0$ this identity is trivial, since $P_{-1}^{(\alpha, \beta)}(x)=0$. It is straightforward to verify this identity for lower $\ell$ by direct calculation.

Below we will prove the identity (3.11) for an arbitrary positive integer $\ell$ by combining a few elementary relations among the Jacobi polynomials of neighboring degrees $n$ and $n-1$ and neighboring parameters $\alpha \pm 1, \beta \pm 1$.,

$$
\text { Lemma (C) } 2(\alpha-1) P_{n}^{(\alpha-1, \beta)}(x)-(n+\alpha+\beta)(1-x) P_{n-1}^{(\alpha, \beta+1)}(x)=2(n+\alpha-1) P_{n}^{(\alpha-2, \beta+1)}(x) \text {, }
$$


Lemma (D) $2(\beta+1) P_{n}^{(\alpha-1, \beta+1)}(x)+(n+\alpha+\beta+1)(1+x) P_{n-1}^{(\alpha, \beta+2)}(x)=2(n+\beta+1) P_{n}^{(\alpha, \beta)}(x)$.

It is straightforward to demonstrate Lemma $(\mathrm{C})$ by using the expansion formula for the Jacobi polynomials (2.38). By using the property $P_{n}^{(\alpha, \beta)}(-x)=(-1)^{n} P_{n}^{(\beta, \alpha)}(x)$, Lemma (D) is obtained from Lemma (C) with the replacements $x \rightarrow-x, \alpha \rightarrow \beta+2, \beta \rightarrow \alpha-1$. The right hand side of the identity (3.11) can be written as

$$
\begin{aligned}
\text { r.h.s. of }(3.11)= & (1+x) P_{\ell-1}^{(\alpha-1, \beta+3)}(x) P_{\ell}^{(\alpha, \beta)}(x) \\
& \times\left\{2(\alpha-1) P_{\ell}^{(\alpha-1, \beta+1)}(x)-(\ell+\alpha+\beta+1)(1-x) P_{\ell-1}^{(\alpha, \beta+2)}(x)\right\} \\
& +(1-x) P_{\ell-1}^{(\alpha+1, \beta+1)}(x) P_{\ell}^{(\alpha-2, \beta+2)}(x) \\
& \times\left\{2(\beta+1) P_{\ell}^{(\alpha-1, \beta+1)}(x)+(\ell+\alpha+\beta+1)(1+x) P_{\ell-1}^{(\alpha, \beta+2)}(x)\right\} \\
& -2(\alpha(1+x)+(\beta+2)(1-x)) P_{\ell-1}^{(\alpha, \beta+2)}(x) P_{\ell}^{(\alpha, \beta)}(x) P_{\ell}^{(\alpha-2, \beta+2)}(x) \\
= & P_{\ell}^{(\alpha, \beta)}(x) P_{\ell}^{(\alpha-2, \beta+2)}(x) \times\left((1+x)\left\{2(\ell+\alpha-1) P_{\ell-1}^{(\alpha-1, \beta+3)}(x)-2 \alpha P_{\ell-1}^{(\alpha, \beta+2)}(x)\right\}\right. \\
& \left.+(1-x)\left\{2(\ell+\beta+1) P_{\ell-1}^{(\alpha+1, \beta+1)}(x)-2(\beta+2) P_{\ell-1}^{(\alpha, \beta+2)}(x)\right\}\right) \\
= & P_{\ell}^{(\alpha, \beta)}(x) P_{\ell}^{(\alpha-2, \beta+2)}(x) P_{\ell-2}^{(\alpha+1, \beta+3)}(x) \\
& \times(\ell+\alpha+\beta+2)((1+x)(x-1)+(1-x)(x+1))=0 .
\end{aligned}
$$

Here we have used Lemma (C) and Lemma (D) in the curly brackets $\{\cdots\}$. This concludes the proof of the identity (3.11).

It is well known that the Laguerre polynomial is obtained from the Jacobi polynomial in the following limit:

$$
\lim _{\beta \rightarrow \infty} P_{n}^{(\alpha, \beta)}\left(1-\frac{2 x}{\beta}\right)=L_{n}^{(\alpha)}(x) .
$$

When the same limit is applied, the Lemma (C) and Lemma (D) reduce to Lemma (A) and Lemma (B). Likewise the cubic identity for the shape invariance of $X_{\ell}$ Jacobi polynomial (3.11) reduces to that of the $X_{\ell}$ Laguerre polynomial (3.4), when divided by -4 and $\alpha$ is replaced by $\alpha+1$.

\section{Hyperbolic DPT}

Here we briefly remark that the shape invariance condition for the deformed hyperbolic DPT reduces, as expected, to the same identity as that for the trigonometric DPT (3.11) derived above. We fix $\ell$ and use new parameters $\alpha$ and $\beta$ instead of $g$ and $h$,

$$
\alpha=-g-\ell-\frac{1}{2}, \quad \beta=-h+\ell-\frac{3}{2} .
$$

By using (3.9), the polynomial $\xi_{\ell}$ and its derivative are expressed just same as (3.10). The conditions for the shape invariance (2.16) with (2.44), after replacing $\eta(x)$ with $x$ and dividing by $\ell+\alpha+\beta+1$, are simplified to the same identity (3.11) as that for the trigonometric DPT.

\section{SUMMARY AND COMMENTS}

Analytic proofs are provided for the shape invariance of the recently derived infinite family of potentials ${ }^{1}$ obtained by deforming the radial oscillator potential and the trigonometric/hyperbolic DPT potential ${ }^{5,6}$ by a degree $\ell(\ell=1,2, \ldots)$ eigenpolynomial. The shape invariance conditions are reduced to new polynomial identities of degree $3 \ell$ involving cubic products of the Laguerre (3.4) or Jacobi polynomials (3.11). Then these identities are proven elementarily by combining simple 
linear identities (3.5) and (3.6) among the Laguerre and (3.12) and (3.13) among the Jacobi, polynomials of neighboring degrees $n, n-1$, and of neighboring parameters, $\alpha, \alpha \pm 1$ and $\beta, \beta \pm 1$. Even these linear identities seem not widely recognized.

The totality of the eigenvalues and the corresponding eigenfunctions together with the normalization constants, etc., of these infinite family of quantum mechanical systems are obtained via the Rodrigues-type formulas (2.7) and (2.8) and reported as (17)-(21), (29)-(36), and (44)-(46) of Ref. 1. In a future publication, ${ }^{15}$ we will present analytic derivation of various results reported in Ref. 1. They include derivation of equivalent but much simpler looking forms of the $X_{\ell}$ polynomials together with the normalization constants, the verification of the actions of the forward and backward shift operators on the $X_{\ell}$ polynomials, Gram-Schmidt orthonormalization for the algebraic construction of the $X_{\ell}$ polynomials, the analysis of the second order differential equations for the $X_{\ell}$ polynomials within the framework of the Fuchsian differential equations in the entire complex $x$-plane, etc. In these analysis, the linear identities Lemma (A) to Lemma (D) play important rôles. The forward and backward shift relations mentioned above [Eqs. (49), (22), (37), and (47) of Ref. 1) are also reduced to polynomial identities involving cubic products of the Laguerre or Jacobi polynomials. We will provide proofs for them in the future publication, ${ }^{15}$ too.

Let us also mention that the same method, deformation in terms of a degree $\ell$ eigenpolynomial, applied to the discrete quantum mechanical Hamiltonians for the Wilson and Askey-Wilson polynomials produced two sets of infinitely many shape-invariant systems together with exceptional $\left(X_{\ell}\right)$ Wilson and Askey-Wilson polynomials $(\ell=1,2, \ldots){ }^{16}$

\section{ACKNOWLEDGMENTS}

This work is supported in part by Grants-in-Aid for Scientific Research from the Ministry of Education, Culture, Sports, Science and Technology, Grant No. 19540179.

${ }^{1}$ S. Odake and R. Sasaki, Phys. Lett. B 679, 414 (2009); e-print arXiv:0906.0142.

${ }^{2}$ L. E. Gendenshtein, JETP Lett. 38, 356 (1983).

${ }^{3}$ L. Infeld and T. E. Hull, Rev. Mod. Phys. 23, 21 (1951).

${ }^{4}$ See, for example, a review, F. Cooper, A. Khare, and U. Sukhatme, Phys. Rep. 251, 267 (1995).

${ }^{5}$ G. Darboux, Leçons sur la Théorie des Surfaces, 2nd ed. (Gauthier-Villars, Paris, 1915), Vol. 2, pp. 210-215.

${ }^{6}$ G. Pöschl and E. Teller, Z. Phys. 83, 143 (1933).

${ }^{7}$ D. Gómez-Ullate, N. Kamran, and R. Milson, J. Approx. Theory, e-print arXiv:0805.3376; J. Math. Anal. Appl. 359, 352 (2009); e-print arXiv:0807.3939.

${ }^{8}$ D. Gómez-Ullate, N. Kamran, and R. Milson, J. Phys. A 37, 10065 (2004).

${ }^{9}$ C. Quesne, J. Phys. A 41, 392001 (2008); e-print arXiv:0807.4087; Symmetry, Integr. Geom.: Methods Appl. 5, 084 (2009); e-print arXiv:0906.2331.

${ }^{10}$ B. Bagchi, C. Quesne, and R. Roychoudhury, Pramana, J. Phys. 73, 337 (2009); e-print arXiv:0812.1488.

${ }^{11}$ S. Bochner, Math. Z. 29, 730 (1929).

${ }^{12}$ M. M. Crum, Q. J. Math. 6, 121 (1955); e-print arXiv:physics/9908019.

${ }^{13}$ S. Odake and R. Sasaki, J. Nonlinear Math. Phys. 12, 507 (2005); e-print arXiv:hep-th/0410102; J. Math. Phys. 49, 053503 (2008); e-print arXiv:0712.4106; Prog. Theor. Phys. 119, 663 (2008); e-print arXiv:0802.1075.

${ }^{14}$ S. Odake and R. Sasaki, J. Math. Phys. 47, 102102 (2006); e-print arXiv:quant-ph/0605215; Phys. Lett. B 641, 112 (2006); e-print arXiv:quant-ph/0605221.

${ }^{15}$ C.-L. Ho, S. Odake, and R. Sasaki, e-print arXiv:0912.5447.

${ }^{16}$ S. Odake and R. Sasaki, Phys. Lett. B 682, 130 (2009); e-print arXiv:0909.3668. 